\title{
Inactivation of USP14 Perturbs Ubiquitin Homeostasis and Delays the Cell Cycle in Mouse Embryonic Fibroblasts and in Fruit Fly Drosophila
}

\author{
Jung Hoon Lee ${ }^{a, b}$ Seoyoung Park ${ }^{a, b}$ Yejin Yun ${ }^{a, c}$ Won Hoon Choi ${ }^{a, c}$ Min-Ji Kang ${ }^{d}$ \\ Min Jae Lee $e^{a, b, c}$ \\ aDepartment of Biochemistry and Molecular Biology, Seoul National University College of Medicine, \\ Seoul, beuroscience Research Institute, Seoul National University College of Medicine, Seoul, \\ 'Department of Biomedical Sciences, Seoul National University Graduate School, Seoul, dDepartment of \\ Biomedical Sciences, Asan Medical Center, University of Ulsan College of Medicine, Seoul, Korea
}

\section{Key Words}

Usp14 • Deubiquitinating enzyme $\cdot$ Ubiquitin $\cdot$ Cell cycle $・$ Drosophila

\begin{abstract}
Background/Aims: The 26S proteasome is the key proteolytic complex for recognition and degradation of polyubiquitinated target substrates in eukaryotes. Among numerous proteasome-associated proteins, a deubiquitinating enzyme (DUB) USP14 has been identified as an endogenous inhibitor of the proteasome. Here, we explored the complex regulatory functions of USP14 that involve ubiquitin (Ub) homeostasis and substrate degradation in flies and mammals. Methods: USP14-null primary and immortalized mouse embryonic fibroblasts (MEFs) and USP14 knocked-down Drosophila were analyzed in this study. We measured proteasome and DUB activities using fluorogenic reporter substrates and adductforming probes. To examine the levels of ubiquitin, we performed immunoblotting and immunohistochemistry. Mass spectrometry (MS) was used to examine polyUb chain linkages and USP14-interacing proteins. Cell cycle was analyzed by flow cytometry, BrdU labeling, and phospho-histone H3 staining. Results: The homeostasis of Ub in USP14 ${ }^{-/-}$MEFs was markedly perturbed because of facilitated clearance of Ub. This phenomenon was recapitulated in muscles of USP14-deficient Drosophila with old ages. Absolute quantitation using MS also revealed that USP14 ${ }^{-/-}$MEFs contained significantly increased amounts of Ub, compared with wild-type. The key phenotype of USP14 $4^{--}$MEFs was their delayed proliferation originated from prolonged interphase possibly through aberrant degradation of cyclins A and B1. We found that knocking down USP14 in Drosophila resulted in delayed eye development associated with reduced mitotic activity. Conclusion: Our study identifies novel cellular functions of USP14 not

J. H. Lee, S. Park and Y. Yun contributed equally to this work.

Min-Ji Kang

and Min Jae Lee

Department of Biomedical Sciences, Asan Medical Center, University of Ulsan College of Medicine

Seoul 05505 (Korea)

E-Mail mjkang@amc.seoul.kr; minjlee@snu.ac.kr
\end{abstract}

KARGER 


\section{Cellular Physiology Cell Physiol Biochem 2018;47:67-82 \\ and Biochemistry Published online: May 15, $2018 \quad \begin{aligned} & \text { DOI: 10.1159/000489750 } \\ & \begin{array}{l}\text { C) } 2018 \text { The Author(s). Published by S. Karger AG, Basel } \\ \text { www.karger.com/cpb }\end{array}\end{aligned}$ \\ Lee et al.: Cellular and Biochemical Roles of USP14}

only in cellular Ub hometostasis but also in cell cycle progression. USP14 was also essential for proper Drosophila eye development. These results strongly suggest that the USP14-mediated proteasome activity regulation may be directly related to various human diseases including cancer.

\section{Introduction}

The ubiquitin-proteasome system (UPS) is a major degradation mechanism in eukaryotic cells that regulates the levels of many short-lived proteins in the cytoplasm and nucleus. Target substrates of the UPS are covalently conjugated with ubiquitin (Ub) through isopeptide bonds between their primary amine group of an internal lysine (Lys) residue(s) and the C-terminal carboxylic acid of Ub. Polyubiquitin (polyUb) chains grown on the Lys residue of Ub moieties are recognized by Ub receptors, some of which are integral subunits of the 26 S proteasome, e.g., RPN1, RPN10, and RPN13, and some are reversibly associated with the proteasome $[1,2]$. Because Ub can form a polymer via one of seven Lys residues (Lys6, Lys11, Lys27, Lys29, Lys33, Lys48, and Lys63) and the amino-terminal amine group, there are eight linkage types of polyUb chain $[3,4]$. Normally multiple types of linkages are used in a polyUb substrate $[5,6]$, and the canonical chain (a Lys48-linked) are usually recognized by the proteasome for degradation. PolyUb chains linked with Lys63 are often recognized by autophagic receptors such as p62/SQSTM1 for their conjugates to be degraded by lysosomes. This mechanism consists of a part of complicated crosstalks between the UPS and autophagy $[7,8]$. On the proteasome, the polyUb chains of the substrate are deubiquitinated by a set of proteasomal deubiquitinating (DUB) enzymes-USP14, UCHL5/UCH37, and RPN11before the substrates are translocated into the catalytic cavity for proteolysis $[9,10]$.

While RPN11, a constitutive subunit of the proteasomal regulatory particle, is located near the substrate entry pore, USP14 and UCHL5 reside relatively far from the entry pore and from Ub receptors, mainly mediating stepwise disassembly of polyUb [11]. This catalytic inhibition or the "chain-trimming" effect may prevent erroneous protein degradation by weakening the interaction between the proteasome and the polyUb chain of a substrate. Moreover, the DUB activity is needed in the proteasome to prevent uncontrolled degradation of $\mathrm{Ub}$ moieties conjugated to target substrates, which will be detrimental to protein homeostasis (proteostasis) in the cell. These DUB enzymes residing on the proteasome belong to distinct classes: USP14 to the USP family, UCHL5 to UCH, and RPN11 to the JAMM family. It is possible that these DUB enzymes have different substrate preferences depending on their specificity for the type of linkage in the polyUb chain; however, polyUb chains composed of different Ub linkages showed only comparable affinity for the $26 \mathrm{~S}$ proteasome and were similarly disassembled by proteasomal DUBs $[12,13]$.

A genetic knockdown of Ubp6, the ortholog of mammalian USP14, accelerates degradation of various target substrates [11], suggesting that USP14 may be a potential therapeutic target in diseases involving accumulation of toxic proteins, such as Alzheimer's and Parkinson's diseases $[14,15]$. The noncatalytic inhibition of the proteasome by USP14 is mediated by the direct interaction with the ATPase ring of the proteasome; this process results in strong interference with RPN11 function and with a conformational change of the proteasome for proper substrate translocation [16]. USP14 is also involved in the "gating" system which is controlled by various cellular factors including metal ions [17-19]. Therefore, mammalian proteasomes function under tonic inhibition by USP14, which expected to be crucial as a secondary quality control mechanism for intracellular proteins. However, the biological functions of these DUB enzymes are still poorly understood.

In the present study, we used primary and immortalized USP $14^{-/-}$mouse embryonic fibroblasts (MEFs) to dissect the biochemical and physiological functions of USP14, which are further characterized in vivo using fruit fly Drosophila melanogaster. As shown below, USP14 ${ }^{-/-}$MEFs showed elevation of both proteasome activity and cellular DUB activity. The mutant MEFs and transgenic flies lacking USP14 also harbor perturbed Ub homeostasis 


\section{Cellular Physiology Cell Physiol Biochem 2018;47:67-82 \begin{tabular}{l|l|l} 
and Biochemistry 10.1159/000489750 & $\begin{array}{l}\text { D } 2018 \text { The Author(s). Published by S. Karger AG, Basel } \\
\text { www.karger.com/cpb }\end{array}$
\end{tabular} \\ Lee et al.: Cellular and Biochemical Roles of USP14}

with upregulated polyUb and reduced free-Ub levels. In addition, we observed delayed cell proliferation and the G2-M checkpoint along with increased amounts of cyclins A and B1. Essential USP14 functions on cell proliferation appeared to be required for normal Drosophila eye development. The abnormality shown here are likely to be related to the perturbed proteasomal activity after the loss of USP14 in MEFs. Thus, these data may help to elucidate complex regulatory functions of USP14 in the proteasome that involve Ub homeostasis and degradation of diverse target substrates in flies and mammals.

\section{Materials and Methods}

\section{Measurement of proteasome and DUB activities}

Hydrolysis of the fluorogenic substrate N-succinyl-Leu-Leu-Val-Tyr-7-amidomethylcoumarin (suc-LLVY-AMC, Enzo Life Sciences, BML-P802) was measured to determine the proteolytic activity of chymotrypsinlike sites of proteasomes, which typically represents total proteasomal activity [20]. Briefly, $10 \mu \mathrm{g}$ of WCEs from WT and USP14-/- MEFs were mixed with $12.5 \mu \mathrm{M}$ suc-LLVY-AMC in a buffer consisting of 50 mM Tris-HCl (pH 7.5), 1 mM EDTA (Daejung Chemicals, 4000-4405), 1 mg/mL BSA (Bovogen Biologicals, BSA025), $1 \mathrm{mM}$ ATP (Biobasic, AB0020), and $1 \mathrm{mM}$ dithiothreitol (DTT; Geogiachem, CDT10). The Ub-AMC hydrolysis reaction was carried out in WCEs. Proteasomal activity and DUB activity were monitored by measuring free AMC fluorescence in a black 96-well plate on a TECAN infinite m200 fluorometer (TECAN, Männedorf, Switzerland). The data were normalized to the basal activity, which was determined by the treatment with a proteasome inhibitor cocktail ( $20 \mu \mathrm{M}$ MG132 plus $5 \mu \mathrm{M}$ epoxomicin).

\section{Immunoblotting (IB)}

WCEs from cells were prepared in RIPA buffer and were used for IB. Proteins were separated by SDS-PAGE and transferred to a polyvinylidene difluoride (PVDF) membrane (Merck Millipore, Darmstadt, Germany). The membranes were blocked with 5\% nonfat milk and probed with the following antibodies: anti-RPN13 (PW9910; Enzo Life Science), anti-RPT2 (PW5030, Enzo), anti-RPN10 (PW9250, Enzo), antiRPT1 (PW8315, Enzo), anti- $\alpha 3$ (PW8115, Enzo), anti- $\alpha 7$ (PW8110; Enzo), anti-HA (12CA5, Roche), antiUSP14 (A300-920A, Bethyl Laboratories), anti-UCHL5 (ab124931, Abcam), anti-Ub (clone P4D1, Santa Cruz Biotechnology, Dallas, TX, USA), anti-p53 (R-19, Santa Cruz Biotechnology), anti-cyclin A (clone B-8, Santa Cruz Biotechnology), anti-cyclin B1 (GNS1, Santa Cruz Biotechnology), and an anti-ACTB antibody (A1978, Sigma). The membranes were then incubated with a horseradish peroxidase-conjugated anti-mouse IgG antibody (81-6720, Invitrogen) or anti-rabbit IgG antibody (G21234, Invitrogen) and visualized using the ECL system (Thermo Fisher Scientific).

\section{Native gel electrophoresis}

Native gel analysis using purified proteasomes or whole cell lysate analysis was performed largely as previously described [15]. Samples were resolved by $3.5 \%$ nondenaturing PAGE for 850 volt-hours and proteasomes were visualized using the fluorogenic substrate suc-LLVY-AMC (Bachem). After the in-gel sucLLVY-AMC hydrolysis assay, proteins in the gel were transferred to PVDF and subsequently immunoblotted against a $\mathrm{CP}$ subunit such as $\beta 1$.

\section{Labeling of DUB enzymes using HA-tagged Ub-vinylmethylester (HA-Ub-VME)}

WCEs $(1.5 \mathrm{mg}$ ) were incubated with an appropriate amount (final concentration: 1 or $4 \mu \mathrm{M}$ ) of HAUb-VME (UW0880, Enzo) in labeling buffer (50 mM Tris- $\mathrm{HCl} \mathrm{pH} \mathrm{7.4,} 5 \mathrm{mM} \mathrm{MgCl}, 0.2 \%$ Tween 20, $2 \mathrm{mM}$ ATP, and $1 \mathrm{mM} \mathrm{DTT}$ ) at $37^{\circ} \mathrm{C}$ for $1 \mathrm{~h}$. The samples were then subjected to either IB analysis with various antibodies or immunoprecipitated with an anti-HA antibody followed with silver staining for visualization and subsequent mass spectrometric analysis.

\section{Silver staining}

For visualization with silver staining, a gel was placed in the fixing solution ( $50 \%$ methanol, $12 \%$ acetic acid) for 90 min with gentle shaking. After that, the gel was washed with $50 \%$ ethanol two times $(2 \times 20$ $\mathrm{min}$ ) and then incubated in the sensitization solution consisting of $0.02 \% \mathrm{Na}_{2} \mathrm{~S}_{2} \mathrm{O}_{3}$ for $1 \mathrm{~min}$. We rinsed the 


\section{Cellular Physiology Cell Physiol Biochem 2018:47:67-82 \begin{tabular}{l|l} 
and Biochemistry Published online: May 15, 2018 & $\begin{array}{l}\text { C } 2018 \text { The Author(s). Published by S. Karger AG, Basel } \\
\text { www.karger.com/cpb }\end{array}$ \\
\hline
\end{tabular}}

Lee et al.: Cellular and Biochemical Roles of USP14

gel three times ( $1 \mathrm{~min}$ each) in water, incubated the gel in the silver nitrate solution $\left(1 \% \mathrm{AgNO}_{3}\right.$ and 0.75 $\mathrm{mL} / \mathrm{L} \mathrm{37 \%} \mathrm{formalin)} \mathrm{for} 20 \mathrm{~min}$. We discarded the silver nitrate solution and rinsed the gel twice in distilled water for $1 \mathrm{~min}$ each time. We developed the silver stain by soaking the gel in the developing solution (6\% $\mathrm{Na}_{2} \mathrm{CO}_{3}, 0.0004 \% \mathrm{Na}_{2} \mathrm{~S}_{2} \mathrm{O}_{3}, 0.5 \mathrm{~mL} / \mathrm{L} 37 \%$ formalin) until bands appeared. The color development was stopped with $1 \%$ acetic acid.

\section{The GST-pulldown assay}

Purified GST or GST-USP14 was incubated with $1.5 \mathrm{mg}$ of WCEs from WT or USP14-/- MEFs in $500 \mu \mathrm{L}$ of GST pulldown buffer (20 mM MOPS, pH 7.2, $25 \mathrm{mM} \beta$-glycerol phosphate, $1 \mathrm{mM} \mathrm{Na}_{3} \mathrm{VO}_{4}, 1 \mathrm{mM}$ DTT, and 1 $\mathrm{mM} \mathrm{CaCl}$ ) containing preequilibrated glutathione-Sepharose $4 \mathrm{~B}$ beads, followed by incubation at $4{ }^{\circ} \mathrm{C}$ with gentle rotation. The beads were then pelleted by centrifugation at $600 \times \mathrm{g}$ for $1 \mathrm{~min}$ and washed five times with $1 \mathrm{~mL}$ of PBS. Proteins bound to the beads were resuspended in $50 \mu \mathrm{L}$ of $2 \times$ sample buffer and analyzed by silver staining.

\section{Mass spectrometric analysis for quantification of Ub linkages}

For absolute quantification of the individual types of polyUb chain in WT and USP14 ${ }^{-/-}$MEFs, three gel regions with species of different molecular weights were subjected to in-gel trypsin digestion and subsequent AQUA mass spectrometric analysis as reported elsewhere [21]. Briefly, isotopically labeled internal standard peptides (Cell Signaling Technology, Danvers, MA, USA) were added to the gel-extracted samples and quantified by amino acid analysis. Data were processed by integrating the appropriate peaks for the native and internal standard peptides, followed by calculation of the ratio of peak areas to estimate the abundance of native peptides. The total Ub amount was determined for a locus as the sum of the modified and unmodified peptide sequence. The amount of mono-Ub (free unconjugated Ub, mono-ubiquitinated proteins, and the endcaps (the terminal Ubs)) are calculated as previously reported [5].

\section{Chase experiments}

To this end, cells were treated with $75 \mu \mathrm{g} / \mathrm{mL}$ cycloheximide, and samples were isolated at chase time points $0,2,4,6,8$, and $10 \mathrm{~h}$ in the absence or presence of proteasome inhibitors $(10 \mu \mathrm{M} \mathrm{MG132}$ and $5 \mu \mathrm{M}$ epoxomicin). Anti-Ub, anti-USP14, and anti-ACTB antibodies were used for IB.

\section{Isolation of primary MEFs}

Primary MEFs were isolated from mouse embryos approximately on embryonic day (E) 13.5: before the embryonic death from USP14 inactivation [22, 23]. Briefly, embryonic bodies at E13.5 were minced in the presence of trypsin after removal of the head, liver, internal blood clot, and guts. The tissues were incubated for $10 \mathrm{~min}$ at $37^{\circ} \mathrm{C}$ and briefly spinned down. The pellet was resuspended in DMEM supplemented with $20 \%$ of FBS for cultivation.

\section{Quantitative RT-PCR}

Total RNA from cultured cells was isolated using the TRIzol Reagent (Invitrogen), followed by further purification on RNeasy mini-columns (Qiagen, USA) with on-column DNase I treatment. cDNA samples were prepared by reverse transcription using the AccuPower RT-premix (Bioneer, Korea). Real-time PCR reactions were then run using the Rotor-Gene RG 3000 system (Corbett Research, AU) with diluted cDNA, the SYBR qPCR master mixture (Kapa Biosystems, USA) as the reporter dye, and 10 pmol of gene-specific primers. Thermal cycling conditions were as follows: $95{ }^{\circ} \mathrm{C}$ for $3 \mathrm{~min}$ to allow for enzyme activation, followed by 40 cycles of $95^{\circ} \mathrm{C}$ for $10 \mathrm{~s}, 58^{\circ} \mathrm{C}$ for $15 \mathrm{~s}$, and $72{ }^{\circ} \mathrm{C}$ for $30 \mathrm{~s}$. The level of each mRNA was normalized to that of ACTB, and the values were plotted as mean \pm SD of three independent experiments. Primer sequences were as follows: for ubiquitin B (UBB), forward (5'-GGCAAGCAGCTAGAAGATGG-3') and reverse (5'-TAATAGCCACCCCTCAGACG-3'); for $\alpha 7$, forward (5'-TTTGATGGCACTCCCAGACTC-3') and reverse (5'-CTGGACCACCTCTAACAGTGC-3'); for ACTB, forward (5'-AAGATCCTGACCGAGCGTG-3') and reverse (5'-TCCAGGGAGGAAGAGGATGC-3').

Cell proliferation assay

For counting, cells were harvested using Trypsin-EDTA. After we resuspended the cell pellet in a fresh medium, $50 \mu \mathrm{L}$ of the sample was mixed with $50 \mu \mathrm{L}$ of $0.4 \%$ trypan blue by gentle pipetting, and then $20 \mu \mathrm{L}$ 


\section{Cellular Physiology Cell Physiol Biochem 2018;47:67-82 \begin{tabular}{l|l} 
and Biochemistry Published online: May 15, 2018 & $\begin{array}{l}\text { C } 2018 \text { The Author(s). Published by S. Karger AG, Basel } \\
\text { www.karger.com/cpb }\end{array}$ \\
\hline
\end{tabular}}

Lee et al.: Cellular and Biochemical Roles of USP14

of the mixture was loaded into each chamber of the hemocytometer. Counting was performed in triplicate by one operator under a microscope with a $40 \times$ objective. For cell viability analysis, Ba/F3 cells were treated with imatinib at various concentrations $(0.01$ to $10 \mu \mathrm{M})$ in the absence or presence of IU1 (50 or $100 \mu \mathrm{M}$ ) for $24 \mathrm{~h}$. Then, a 3-(4, 5-dimethylthiazol-2-yl)-2, 5-diphenyltetrazolium bromide (MTT) solution (Sigma) was added to the medium with incubation for $3 \mathrm{~h}$ at $37{ }^{\circ} \mathrm{C}$ in a humidified atmosphere of $95 \%$ air and $5 \%$ $\mathrm{CO}_{2}$ for formation of blue-MTT-formazan products, which were quantified by measuring absorbance (test wavelength $570 \mathrm{~nm}$ and reference wavelength $630 \mathrm{~nm}$ ).

\section{Cell cycle analysis}

At various passage numbers, the cells were collected, washed with cold phosphate-buffered saline (PBS), and fixed in 70\% ethanol at $4{ }^{\circ} \mathrm{C}$ overnight. They were then centrifuged at $2000 \mathrm{rpm}$ for $5 \mathrm{~min}$, resuspended in PBS, and incubated with propidium iodide $(20 \mu \mathrm{g} / \mathrm{mL}$ in PBS with $0.05 \%$ Tween 20$)$ and RNase A (50 $\mu \mathrm{g} / \mathrm{mL}$, Sigma) at room temperature for $30 \mathrm{~min}$ in the dark. The cells were then analyzed by flow cytometry (BD Bioscience).

\section{Quantitative live-cell imaging analysis}

Differences in mitotic duration and interphase between WT and USP14 ${ }^{-/-}$MEFs were measured using automatic live-cell imaging analysis as previously reported [24]. Briefly, MEFs were infected for $8 \mathrm{~h}$ with a retrovirus expressing GFP-H2B. After $24 \mathrm{~h}$, the cells were split into aliquots and seeded in a 96-well plate $(\sim 7,000$ cells per well) and were imaged for $48 \mathrm{~h}$ at 15 -min intervals. Mitotic duration was defined as the difference between the mitotic entry and chromatid segregation.

\section{The cyclin-luciferase activity assay}

Immortalized WT and USP14 ${ }^{-/-}$MEFs were infected with an adenovirus expressing a fusion of $\mathrm{N}$-terminal fragments and promoter regions of cyclin A or cyclin B1 to luciferase [25] for $24 \mathrm{~h}$. WCEs were prepared and subjected to the conventional luciferase assay (Promega). The intensity of luminescence was normalized to total protein content.

\section{Fly strains and experiment}

All Drosophila stocks were raised on a standard cornmeal medium at $25^{\circ} \mathrm{C}$. Genes were expressed in Drosophila by means of the standard Gal4/UAS system [26]. Gmr-gal4 (stock \#1104) and P[Act5CGAL4. Switch.PR] (stock \#9381) were obtained from the Bloomington Stock Center. The UAS-lacZ RNAi fly lines were generously provided by Dr. Masayuki Miura. UAS-USP14 RNAi (stock \#110227) were obtained from the Vienna Drosophila Resource Center. For the induction of genes, $100 \mu \mathrm{l}$ of a $5 \mathrm{mg} / \mathrm{ml}$ solution of RU486 (M4086, Sigma) or vehicle (80\% ethanol) was added on top of food in a vial and dried overnight before being fed to the flies.

\section{Immunohistochemistry and in vivo proliferation assays using Drosophila}

Immunostaining of larvae or indirectflight sections of Drosophila was performed as described previously [27]. For in vivo 5-bromo-2-deoxyuridine (BrdU) assay, the larval eye discs were dissected on Schneiderís Drosophila Medium (Invitrogen, 11720), and then transferred into Schneider's Medium containing 0.25 $\mathrm{mg} / \mathrm{ml} \mathrm{BrdU}$ for $90 \mathrm{~min}$ at room temperature. After washing the samples three times with PBS, they were fixed with 4\% paraformaldehyde in PBS for 15 min. The samples were washed three times in PBS and then incubated with 5 units of DNase I (Promega, M6101) in $95 \mathrm{ul}$ of DNaseI Buffer for $2 \mathrm{~h}$ at $37^{\circ} \mathrm{C}$. The eye discs were washed three times with PBST (1X PBS plus 0.1\% Triton X-100) for $10 \mathrm{~min}$ each and then incubated with mouse anti-BrdU antibody (1:50, BD Pharmingen) overnight at $4{ }^{\circ} \mathrm{C}$. For immunohistochemistry of phospo-histone H3 (pH3), the larvae were dissected in Drosophila Ringer's solution and fixed in 1X PBS with $4 \%$ paraformaldehyde for 30 min. Samples were washed in $0.1 \%$ PBST for $2 \mathrm{~h}$, then incubated with rabbit anti-phospo-histone-H3 antibody (1:2000, Upstate biotechnology) and anti-elav antibody (1:50, Developmental Studies Hybridoma Bank) at $4{ }^{\circ} \mathrm{C}$ overnight. After washing the samples with PBST for 2h, secondary antibodies were incubated for $3 \mathrm{~h}$, then washed in PBST for $2 \mathrm{~h}$. The samples were mounted with 50\% glycerol. For Ub immunostaining, indirect flight muscles were dissected in PBS and fixed for 20 minutes in PBS with 4\% paraformaldehyde and 0.2\% Triton X-100. After washing, samples were incubated overnight with anti-ubiquitinylated protein antibody (1:200, clone, FK2, Enzo, BML-PW8810-0100) and 


\section{Cellular Physiology Cell Physiol Biochem 2018;47:67-82 \begin{tabular}{l|l} 
and Biochemistry Published online: May 15, 2018 & $\begin{array}{l}\text { DO } 2018 \text { The Author(s). Published by S. Karger AG, Basel } \\
\text { www.karger.com/cpb }\end{array}$
\end{tabular} \\ Lee et al.: Cellular and Biochemical Roles of USP14}

secondary antibodies. All fluorescent images were captured on a Zeiss LSM710 confocal microscope under identical conditions.

\section{Results}

USP14 deficiency enhances both proteasomal and DUB enzymatic activity

We have previously determined that yeast Ubp6 and of its mammalian ortholog USP14 function as an endogenous inhibitor of proteasome activity $[9,28]$. To examine the antagonizing effect of USP14 on degradation of various proteasome substrates, we used mouse embryonic fibroblasts (MEFs) because USP14 ${ }^{-/-}$homozygous mutant mice die approximately on embryonic day 13.5 owing to various defects in neuronal development [29]. Recently, the Goldberg group reported that purified proteasomes from USP14 ${ }^{-/-}$MEFs have higher proteolytic activity than those from wild-type (WT) MEFs [30]. We kinetically assessed the chymotrypsin-like peptidase activities in whole-cell extracts (WCEs) of MEFs by means of the fluorogenic proteasome substrate N-succinyl-Leu-Leu-Val-Tyr-7amidomethylcoumarin (Suc-LLVY-AMC). In immortalized MEFs, the fluorescent signals possibly representing overall peptidase activity of the $26 \mathrm{~S}$ proteasome were significantly stronger in USP14 $4^{-/}$MEFs than in WT MEFs (Fig. 1A). Having identified USP14 as a negative regulator of proteasome function, we went on to test whether a small-molecule inhibitor of USP14, as a disinhibitor, might enhance proteasome activity. When we treated MEFs with IU1 (a potent inhibitor of USP14 [28]), the suc-LLCY-AMC hydrolytic activity in WT MEFs was significantly enhanced (Fig. 1B). On the contrary, in USP14 ${ }^{-/-}$MEFs which maintain higher peptidase activity as compared with WT MEFs, IU1 treatment resulted in little or no change, indicating that the small-molecule inhibitor is largely specific to USP14 (Fig. 1B). To examine the consequences of a loss of USP14 for cellular levels of the $26 \mathrm{~S}$ proteasome, we compared the amounts of several subunits of the regulatory particle and core particle and immunoblotting (IB) analysis indicated that proteasomes in both MEF groups were virtually identical (Fig. 1C), thereby indicating that the changes in proteasomal and DUB activities in USP14 ${ }^{-/-}$MEFs do not originate from the changes of proteasome amounts in the cell.

The effects of USP14 inactivation on total cellular DUB activity were examined by hydrolysis of Ub-AMC, a highly sensitive fluorogenic substrate of DUB enzymes. We found that USP14 $4^{-/-}$MEFs harbored higher cellular DUB activity than the WT did (Fig. 1D), possibly because of the compensatory increase in another DUB enzyme activity. To determine which DUB enzymes are responsible for this enhanced DUB activity, we labeled total DUB enzymes with HA-tagged Ub-vinylmethylester (HA-Ub-VME), which specifically reacts with the activesite cysteine in thiol protease DUB enzymes and forms stable adducts (Fig. 1E). When USP14 was covalently modified by Ub-VME, the electrophoretic mobility of USP14 was reduced by $7.5 \mathrm{kDa}$, which corresponds to the size of Ub (Fig. 1F). When the WCEs from USP14 ${ }^{-{ }^{-}}$MEFs were used, in agreement with the Ub-AMC assay data, a couple of Ub-VME-DUB enzyme adduct signals (during IB with an anti-HA antibody) showed stronger intensity than those in WT controls (Fig. 1F). Notably, the levels of both UCHL5 (another reversible DUB enzyme associated with the $26 \mathrm{~S}$ proteasome) and Ub-VME-UCHL5 adducts were higher in USP14 $4^{-/}$ MEFs, consistent with a recent report [30]. Although the underlying molecular mechanism needs to be determined, these results indicate that USP14 deficiency results in an increased steady-state amount of UCHL5 and perhaps increased activity of UCHL5 as well.

Given these findings, we decided to further identify the specific targets of HA-Ub-VME in USP $14^{-/-}$MEFs. For this purpose, we subjected the reaction mixtures to immunoprecipitation with an anti-HA antibody, resolved the precipitated proteins by SDS-PAGE and stained them with silver nitrate (silver staining; Fig. 1G). Five prominent bands, which showed stronger signals in USP14 ${ }^{-/-}$than in WT MEFs, were subjected to tandem mass spectrometry (MS/MS) for identification and turned out to be integral components of the proteasome (i.e., RPN1, RPN13, and RPT2) and known proteasome-interacting proteins (i.e., HUWE1 and HSP70; Fig. 1E). In addition, our GST-pulldown assay using recombinant GST-USP14 and WCEs from both MEF groups, followed by silver staining and mass spectrometric analysis, indicated 


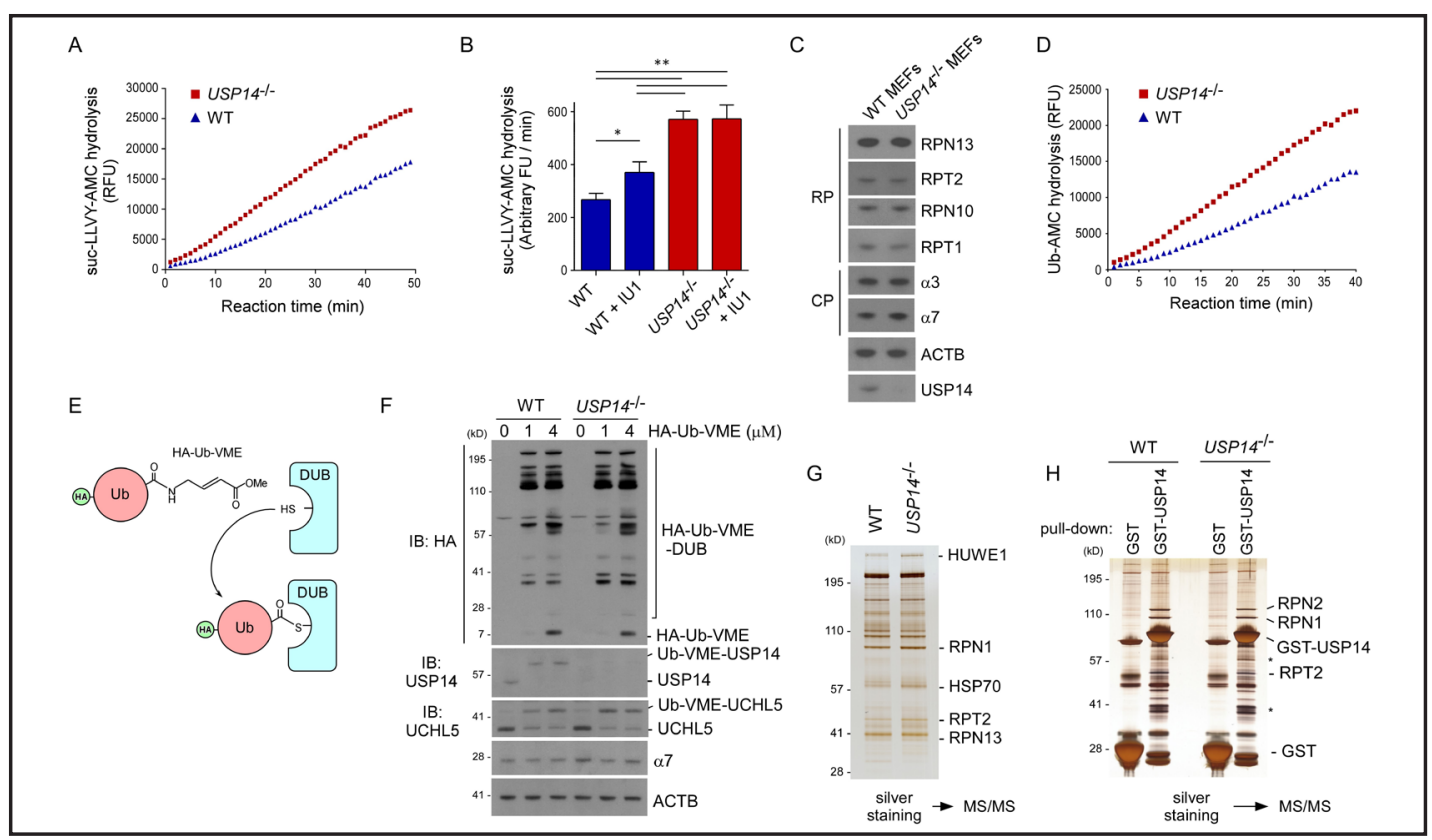

Fig. 1. Deletion of USP14 in mouse embryonic fibroblasts (MEFs) resulted in elevation of both proteasomal and DUB activity. (A) Proteasome activity in wild-type (WT) and USP14-/ MEFs was measured via hydrolysis of the fluorogenic substrate suc-LLVY-AMC using whole-cell extracts (WCEs). Values were normalized to the basal activity, which was determined by the treatment with a proteasome inhibitor cocktail (20 $\mu$ M MG132 plus $5 \mu \mathrm{M}$ epoxomicin) for $4 \mathrm{~h}$. RFU: relative fluorescence units. Similar results were obtained in three independent experiments. (B) Proteasome activity measured by hydrolysis of the fluorogenic substrate sucLLVY-AMC at 20-min of reactions was compared between wild-type (WT) and USP14-/- MEFs after they were treated with the USP14 inhibitor IU1 (50 $\mu \mathrm{M}, 4 \mathrm{~h})$. WT MEFs showed modestly enhanced proteasome activity after inhibition of USP14, while the proteasome activity in USP14 ${ }^{-{ }^{-}}$MEFs was not affected by IU1. Values (arbitrary fluorescence unit/reaction time) were normalized to the basal activity, which was determined by the treatment with a proteasome inhibitor cocktail (20 $\mu \mathrm{M}$ MG132 plus $5 \mu \mathrm{M}$ epoxomicin) for $4 \mathrm{~h}$. The error bars represent SEMs of four samples. ${ }^{*} \mathrm{P}<0.05$, ${ }^{* *} \mathrm{P}<0.01$ from Bonferroni's multiple comparison ANOVA test. (C) Immunoblot (IB) analysis of WCEs from WT and USP14 ${ }^{-/-}$MEFs showed that the amounts of subunits of the proteasome regulatory particle (RP) and core particle (CP) were not significantly changed in USP14 ${ }^{-/-}$MEFs. ACTB/ $\beta$ actin served as a loading control. (D) Total DUB activity was monitored by hydrolysis of Ub-AMC using WCEs from WT and USP14-/ MEFs. (E) Schemes for labeling DUB enzymes using HAtagged Ub-vinylmethylester (HA-Ub-VME) as a probe, which yields a covalent adduct with the active-site Cys residue. (F) WCEs from WT and USP14 ${ }^{-/-}$MEFs were treated with the indicated amounts of HA-Ub-VME for $4 \mathrm{~h}$, and the products were analyzed by SDS-PAGE/IB. UV-VME adducts with USP14 or UCHL5 DUB enzymes were identified by IB analysis with anti-HA, anti-USP14, and anti-UCHL5 antibodies. (G) The same reactions were conducted as in (F), except that the HA-Ub-VME-DUB adducts were first isolated by anti-HA immunoprecipitation and then resolved by SDS-PAGE. Bands were visualized by silver staining and identified by tandem mass spectrometry (MS/MS). (H) A GST pulldown assay to identify USP14-binding proteins. Purified GST or GST-USP14 proteins $(10 \mu \mathrm{g})$ were incubated with $1.5 \mathrm{mg}$ of WCE from WT and USP14 ${ }^{-/-}$MEFs. Samples were analyzed by SDS-PAGE and silver-staining. Specific bands were then analyzed by MS/MS and all were identified as subunits of the proteasomal regulatory particle, including RPN1, RPN2, and RPT2. Asterisks $\left({ }^{*}\right)$ indicate nonspecific signals. This experiment shows that most of USP14 functionally interacts with the regulatory particle of the $26 \mathrm{~S}$ proteasome.

that the major interaction partners of USP14 are also proteasome subunits (Fig. 1H). Only a minor fraction of USP14 were detected as a proteasome-unbound form in crude extracts [31]. These results strongly indicate that the majority of the DUB activity of USP14 is mainly linked to the Ub chain trimming on the proteasome rather than its functionality outside the proteasome context. The activation of USP14 by the proteasome [28] is partly the reason for 


\section{Cellular Physiology Cell Physiol Biochem 2018;47:67-82 and Biochemistry Published online: May 15, $2018 \quad \begin{aligned} & \text { DOI: 10.1159/000489750 } \\ & \begin{array}{l}\text { (c) } 2018 \text { The Author(s). Published by S. Karger AG, Basel } \\ \text { ww.karger.com/cpb }\end{array}\end{aligned}$ Lee et al.: Cellular and Biochemical Roles of USP14}

this connection, but the detailed molecular mechanism has yet to be identified. Together, our data indicate that the enhanced proteasome activity and DUB activity in USP14-null cells are directly related to the inhibitory effect of USP14 on the proteasome.

Assembly of USP14-deficient proteasomes appears to be normal but the core particle (CP) levels are elevated

Since yeast Ubp6 was reported to be critical for the 20S regulatory particle (RP) assembly process [32], we next examined whether the altered proteasome acitivity in USP14null cells originate from the abnormal proteasome assembly or structural integrity of the $26 \mathrm{~S}$ proteasome. On the basis of native gel electrophoresis and in-gel detection of suc-LLVYAMC hydrolysis using whole MEF cell lysates, we observed slightly higher fluorescence signals from the RP-CP (singly-capped) and the $\mathrm{RP}_{2}$-CP (doubly-capped 26S) forms of proteasomes (Fig. $2 \mathrm{~A})$. The gel was then subjected to the immunoblotting against a CP subunit $\beta 1$, which showed no significant defects in proteasome assembly even

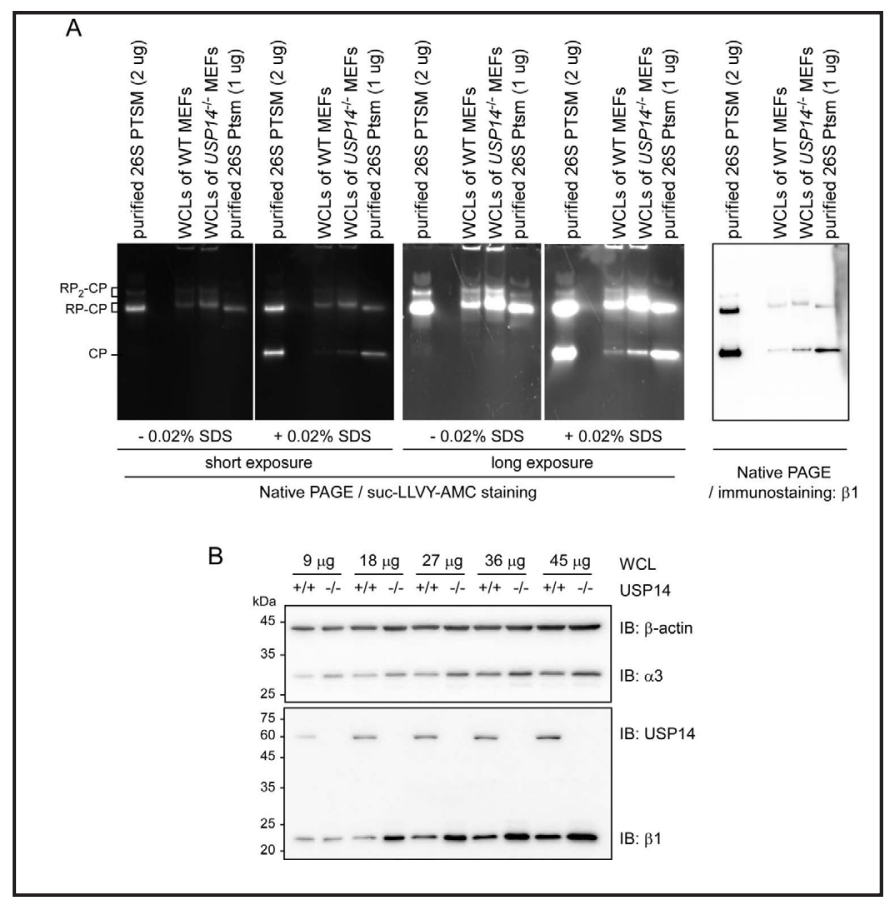

Fig. 2. Assembly and gating of WT and USP $14 \%$ proteasomes are comparable in native PAGE analysis. (A) The composition and activity of proteasomes from WT and USP14\% MEFs were determined by native PAGE. Whole cell extracts from the MEFs were resolved on 3.5\% non-denaturing gels and subjected to in-gel peptidase assays with fluorogenic substrate suc-LLVY-AMC. Purified human proteasomes with indicated amounts were used as controls. The addition of SDS (0.02\%) activated latent CP. (right) Following the activity assays, the native gels were immunoblotted for a CP subunit $\beta 1$. RP, regulatory particle. CP, core particle. (B) Whole cell extracts from WT and USP14 ${ }^{-/} \operatorname{MEFs}(9,18,27,36$, and $45 \mu \mathrm{g}$ ) were subjected to denaturing SDS-PAGE/IB analysis. Anti- $\beta$-actin and anti- $\alpha 3$ antibodies (upper panel) or anti-USP14 and anti- $\beta 1$ antibodies were incubated simultaneously for immunoblotting. though a modest increase of $\mathrm{CP}$ was observed in USP14 $\%$ MEFs. The elevated levels of CP in USP14 $\%$ MEFs were confirmed with a denaturing-condition SDS-PAGE assay using the equivalent amounts of whole cell lysates from MEFs (Fig. 2B). Consistent with this, when we activated the latent CP in the native gels with $0.02 \%$ SDS, the stronger fluorescence signal was detected in the USP14deficient CP than the WT CP (Fig. 2A). This may reflect the role of USP14 in 20S gate opening [33]. Although further work will be required to determine how cellular proteasome biogenesis is controlled by USP14-deficiency, considering the compensatory overexpression of CP subunits, the overall increase of proteasome activity in USP14 $\%$ MEFs is more likely to be linked with the USP14's noncatalytic effects, which regulate the ATP hydrolysis and conformation states of $26 \mathrm{~S}$ proteasomes [10,30].

USP14 deficiency perturbs the recycling of ubiquitin from polyubiquitinated proteins

USP14 is expected to play a critical role in the maintenance of cellular levels of free (unanchored) Ub through stepwise disassembly of the Ub chains from the distal end, thus allowing for recycling of Ub from proteasomal degradation. Accordingly, we tested whether the lack of USP14 affects the half-life and homeostasis of intracellular Ub proteins. The 


\section{Cellular Physiology Cell Physiol Biochem 2018;47:67-82 \begin{tabular}{l|l} 
DOI: 10.1159/000489750 & $\begin{array}{l}\text { O 2018 The Author(s). Published by S. Karger AG, Basel } \\
\text { www.karger.com/cpb }\end{array}$
\end{tabular} Lee et al.: Cellular and Biochemical Roles of USP14}

Fig. 3. The loss of USP14 results in dysfunction of ubiquitin (Ub) homeostasis in MEFs. (A) Total polyubiquitin (poly$\mathrm{Ub}$; $\left.(\mathrm{Ub})_{\mathrm{n}}\right)$ levels in WCEs significantly increased, while free (unanchored) Ub levels decreased, in USP14 ${ }^{-/-}$MEFs relative to WT MEFs. The treatment with proteasome inhibitors (the combination of $10 \mu \mathrm{M}$ MG132 and 5 $\mu \mathrm{M}$ epoxomicin for 4

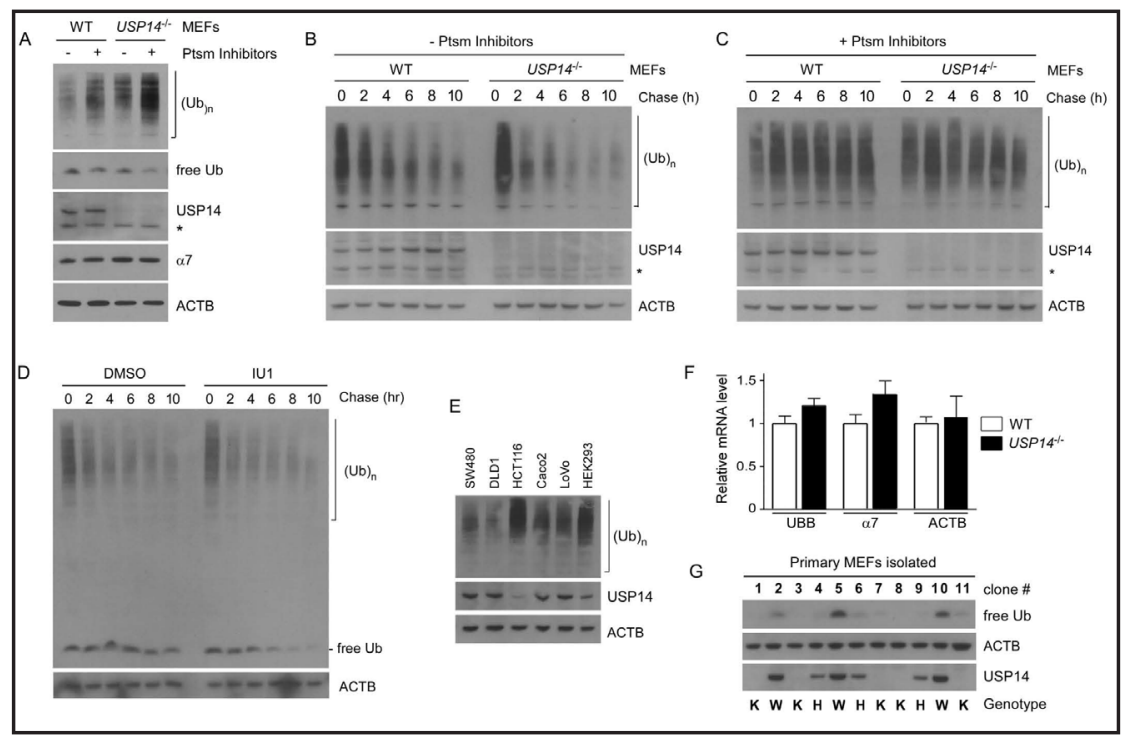
h) further increased these differences. Anti-Ub, anti-USP14, anti- $\alpha 7$, and anti- $\beta$-actin (anti-ACTB) antibodies were used for IB analysis. Asterisks $(*)$ indicate nonspecific IB signals. (B, C) Chase experiments for total polyUb in WT and USP14 ${ }^{-/} \mathrm{MEFs}$ were carried out at the indicated time points after the addition of $75 \mu \mathrm{g} / \mathrm{mL}$ cycloheximide at time zero in the absence (B) or presence (C) of proteasome inhibitors (10 $\mu \mathrm{M}$ MG132 and $5 \mu \mathrm{M}$ epoxomicin added at time zero). (D) IU1, a small-molecule USP14 inhibitor, facilitates the degradation of polyUb and free Ub in HeLa cells. To examine the effect of chemical inhibition of USP14 on the half-life of Ub, we preincubated IU1 $(75 \mu \mathrm{M})$ with HeLa cells, and chase experiments using cycloheximide were conducted at the indicated time points. (E) The correlation between USP14 and polyUb levels was examined in colon cancer cell lines, including SW480, DLD1, HCT116, Caco2, and LoVo cells, along with HEK293 cells. (F) USP14 does not significantly regulate mRNA encoding ubiquitin B (UBB) and $\alpha 7$. Total RNA was isolated from MEFs using TRIzol extraction and RNeasy column purification methods, and quantitative RT-PCR was carried out using primers specific for UBB, $\alpha 7$, and ACTB (control). The values are presented as mean $\pm \mathrm{SD}$ of three independent experiments. (G) An unbiased experiment to examine the USP14 genotype and free-Ub levels in MEFs. Primary MEFs were isolated from embryos on embryonic day 13.5 after mating of heterozygous (USP14 $4^{+-}$) mutants. Eleven clones (\#1 to \#11) were obtained and blindly analyzed for the levels of free Ub and USP14 by IB. Genotype analysis was independently performed using genomic DNA from placental membranes.

simultaneous treatment with proteasome inhibitors MG132 and PS341 caused accumulation of polyUb-conjugated proteins in both WT and USP14 $4^{--}$MEFs (Fig. 3A). We, however, also found that USP14 ${ }^{-/-}$MEFs contain increased levels of polyubiquitinated proteins under normal conditions and that the polyUb levels were further increased by treatment with the proteasome inhibitors. This is mainly because that the cells lacking USP14 have more amounts of Ub (Fig. 5) through the adaptive response to the Ub stress [34]. On the contrary, amounts of free $\mathrm{Ub}$, which more adequetly represent the available $\mathrm{Ub}$ pool in the cell, were significantly lower in USP14 $4^{--}$MEFs than in WT MEFs. Overall, the changes in the levels of free $\mathrm{Ub}$ and polyUb conjugates in USP14 $4^{-/-} \mathrm{MEFs}$ appear to be the consequences of complicated effects resulted from elevated cellular Ub amounts, conformational changes of proteasome holoenzymes, lack of processivity of proteasomal USP14 (reduced Ub chaintrimming effect), and accelerated proteasomal degradation of polyUb conjugates in the cell, possibly including Ub moiety itself.

The effect of USP14 deficiency on Ub proteostasis was further examined by a cycloheximide (CHX) chase assay combined with IB analysis. The polyUb-conjugated protein amounts in the CHX chase experiment decreased more rapidly in USP14 ${ }^{-/-}$MEFs than in the WT although, at the initial time point (at chase time 0), polyUb amounts were higher 


\section{Cellular Physiology Cell Physiol Biochem 2018;47:67-82 and Biochemistry Published online: May 15, $2018 \quad$\begin{tabular}{l|l} 
DOI: 10.1159/000489750 2018 The Author(s). Published by S. Karger AG, Basel \\
www.karger.com/cpb
\end{tabular} Lee et al.: Cellular and Biochemical Roles of USP14}

in the USP14 knockout MEFs (Fig. 3B). In the presence of proteasome inhibitors, levels of polyUb conjugates were virtually constant in WT up to the chase time point $10 \mathrm{~h}$, while in USP14 $4^{-1-}$ MEFs they began to decrease at $\sim 6 \mathrm{~h}$ after CHX treatment (Fig. 3C). In line with this finding, the chase experiment in the presence of a USP14 inhibitor, IU1 (75 $\mu \mathrm{M})$, also showed accelerated depletion of both polyUb conjugates and free Ub pool in HeLa cells (Fig. 3D). Taken together, our data indicate that not only genetic inactivation of USP14 but also chemical inhibition of USP14 may increase the rate of proteasomal degradation of Ub. It will be interesting to further dissect the consequences of this Ub stress for the cell on a global level and possible pathological implications of this destructive phenomenon.

We have previously shown that USP14 levels strongly correlate with $\beta$-cateninmediated colon cancer development [35]. Among colon cancer cell lines, SW480, LOVO, DLD1, and Caco2 colon cancer cells have significantly or modestly increased levels of USP14 in contrast to HEK293 cells or HCT116 cells, which have lost USP14 expression because of somatic mutations [35]. Using HCT116 cells and other colon cancer cells, we next tested whether the expression magnitude of USP14 correlates with polyUb amounts and found that HCT116 cells, which show the weakest USP14 expression, contain markedly increased polyUb levels (Fig. 3E). The mRNA levels of ubiquitin B (UBB) in USP14 ${ }^{-/-}$MEFs were not significantly different to those in the WT (Fig. 3F), suggesting that the positive effect of USP14 inactivation on the amounts of polyUb conjugates involves post-translational events, instead of transcriptional alteration. Finally, we conducted a blinded experiment on primary MEFs of unknown genotypes. We established 11 primary MEF cell lines from the progeny of heterozygous (USP14 ${ }^{+/-}$) mating. The independent PCR-based genotyping using placental DNA along with unbiased IB analysis of the MEFs clearly indicated that the amount of USP14 mostly correlated with the levels of free Ub in primary MEFs (Fig. 3G). These data suggest that USP14's Ub chain-trimming effect is necessary to maintain the pool of available Ub units in the cell.

We then tried to validate the upregulation of Ub-conjugated species in vivo by using a transgenic Drosophila model, which inducibly expresses USP14 siRNA in muscles after RU486 treatment. Knockdown of USP14 did not affect the overall muscle differentiation (as estimated by sarcomere assembly) or Ub-conjugates levels (by immunostaining using the FK2 clone antibody) in 1-week old flies (Fig. 4A and $4 A^{\prime}$ ). In 5-week old flies, we observed accumulation of ubiquitinated proteins, which may reflect the defected protein homeostasis connected with the age-related deposition of protein aggregates [36]. Knocking down USP14 by feeding RU486 (Act5C $\mathrm{C}^{\mathrm{GS}}>$ usp14 RNAi RU486+) dramatically increased the level of Ubconjugates-associated protein aggregates in muscles of old flies, compared with controls (Act5C ${ }^{\mathrm{GS}}>$ usp14 RNAi RU486; Fig. 4B and 4B'), suggesting that USP14 is necessary for proper proteostasis in muscles and possibly the aging process as well. Our results strongly

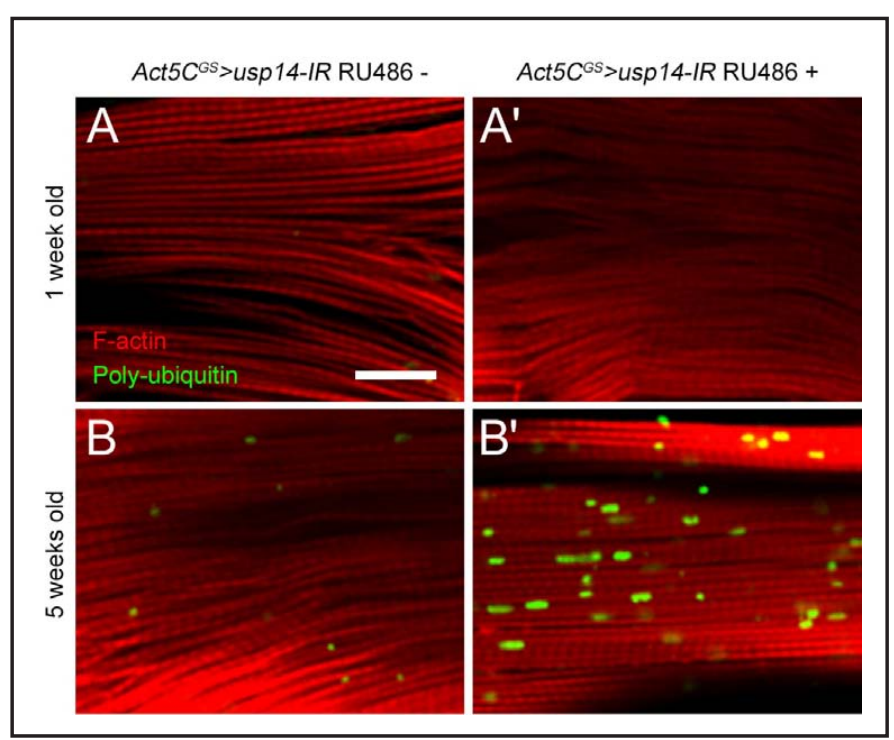

Fig. 4. Knocking down USP14 in Drosophila melanogaster results in accumulated polyUb conjugates. Transgenic Drosophila models inducibly expressing USP14-interfering-siRNA (USP14-IR) in nonflying muscles were treated with control (80\% ethanol; $A$ and $B$ ) and RU486 ( $A^{\prime}$ and $B^{\prime}$ ) for $7 \mathrm{~d}$ at different ages. A and A', 1 week-old. $B$ and B', 5-week old flies. Scale bar, $20 \mu \mathrm{m}$. 
suggest that upregulation of proteasomal activity through USP14 nullification leads to faciliated degradation of poly-Ub conjugates but results in increased steady-states of total Ub levels in MEFs. Therefore, a blockage of USP14 activity may affect not only the proteasomal degradation of polyUb substrates but also the Ub biogenesis at the transctiorional step as well.

Absolute quantification (AQUA) mass spectrometric analysis of global dynamics of Ub linkages in USP14\% MEFS

USP14 deubiquitinase progressively trims polyUb in a substrate from the distal end of the chain. To analyze and quantify Ub species that are accumulated in USP14 $4^{--} \mathrm{MEFs}$, we used AQUA-based mass spectrometry, which allows for precise quantification of tryptic peptides derived from total Ub and Ub-Ub chain linkages including major chains (based on either the K48 or K63 bond) and minor chains based on the K6, K11, K27, or K33 linkage. Significant upregulation of mono-Ub (both free unconjugated $\mathrm{Ub}$ and mono-ubiquitinated proteins; see Methods for details) was observed in USP14 ${ }^{-/-}$MEFs compared with WT MEFs (669.8 fmol in WT MEFs vs. $871 \mathrm{fmol}$ in USP14 ${ }^{-/-}$MEFs; Fig. 5A). Amounts of all detectable Ub isopeptide species, such as K48 (337.9 vs. $479.4 \mathrm{fmol}$ ), K63 (97.8 vs. $138.6 \mathrm{fmol}$ ), K11 ( 90.8 vs. $109.2 \mathrm{fmol}$ ), K33 (28.6 vs. $70.6 \mathrm{fmol}$ ), K27 (0.8 vs. $27.3 \mathrm{fmol}$ ), and K6 (9.1 fmol in WT MEFs vs. $20.7 \mathrm{fmol}$ in USP14 ${ }^{-/-}$MEFs), significantly increased in USP14 $4^{-/-}$MEFs (Fig. 5A). These quantitative proteomic data were validated by IB analysis using K48- and K63linkage-specific antibodies (Fig. 5B). Consistent with the AQUA-MS analysis, the levels of these polyUb species were significantly higher in USP14 $14^{-/}$MEFs, while proteasome subunit RPT5 levels were unchanged (Fig. 5B). Despite the overall increases in total Ub amounts, the proportion of each polyUb chain linkage in USP14 ${ }^{-/-}$MEFs was largely unchanged (Fig. 5C). These results indicated that USP14 has a broad DUB specificity toward various polyUb chain linkages as previously reported $[13,37]$, not having a preference for a specific type of chain linkages.

Fig. 5. Absolute quantific a tion (AQUA) mass spectrometry revealed that the amounts of polyUb chains of some Ub linkage types were elevated in USP14 ${ }^{-/-}$ MEFs. (A) Absolute amounts (fmol) of distinct polyUb chains in WCEs of WT and USP14 ${ }^{-/-}$ MEFs. (B) IB analysis using specific antibodies recognizing Lys48- or Lys63-linked polyUb chains. (C) Same as in (A), except the proportion (percent of total Ub) of each polyUb linkage is shown. (D) Samples for AQUA mass spectrometry were separated into three groups by size. (E) Absolute amounts (fmol) of distinct polyUb chains from different regions of WT and USP14 $4^{-/-}$MEF samples. (F) Same as in (E) except that the proportion of a specific linkage in total Ub is shown. Amounts of polyUb chains of most linkage types in all regions are relatively similar between WT and USP14 ${ }^{-/-}$MEFs, whereas significantly increased amounts of Lys48-linked polyUb chains and decreased monoUb levels were observed in samples from region 3.
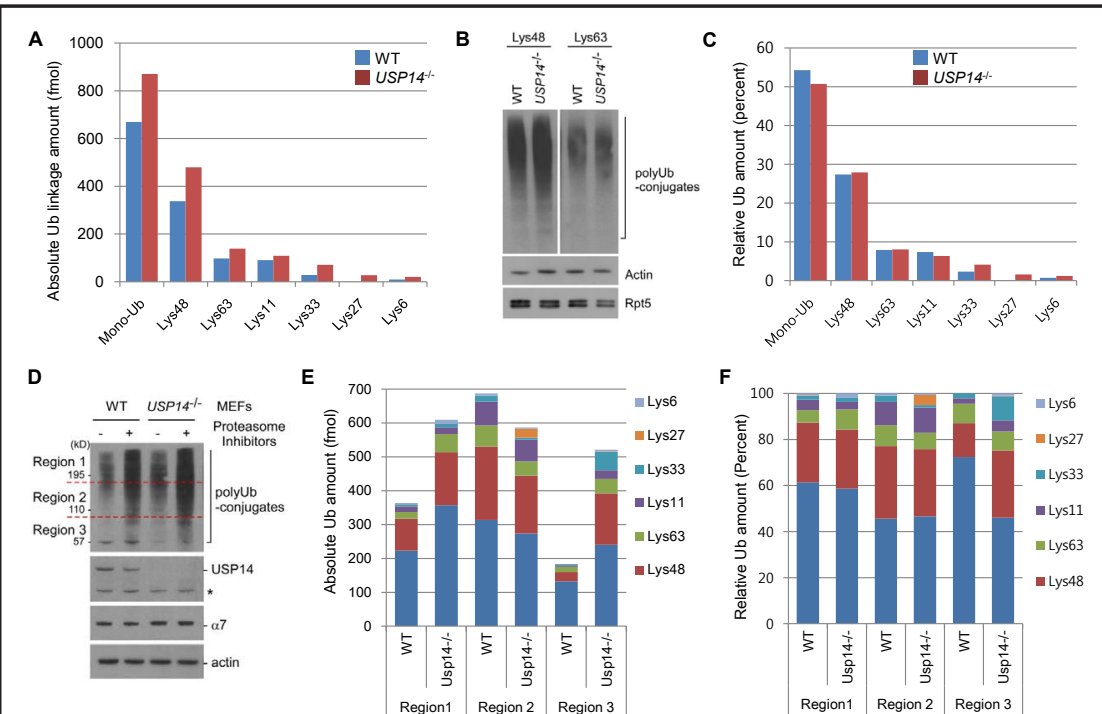


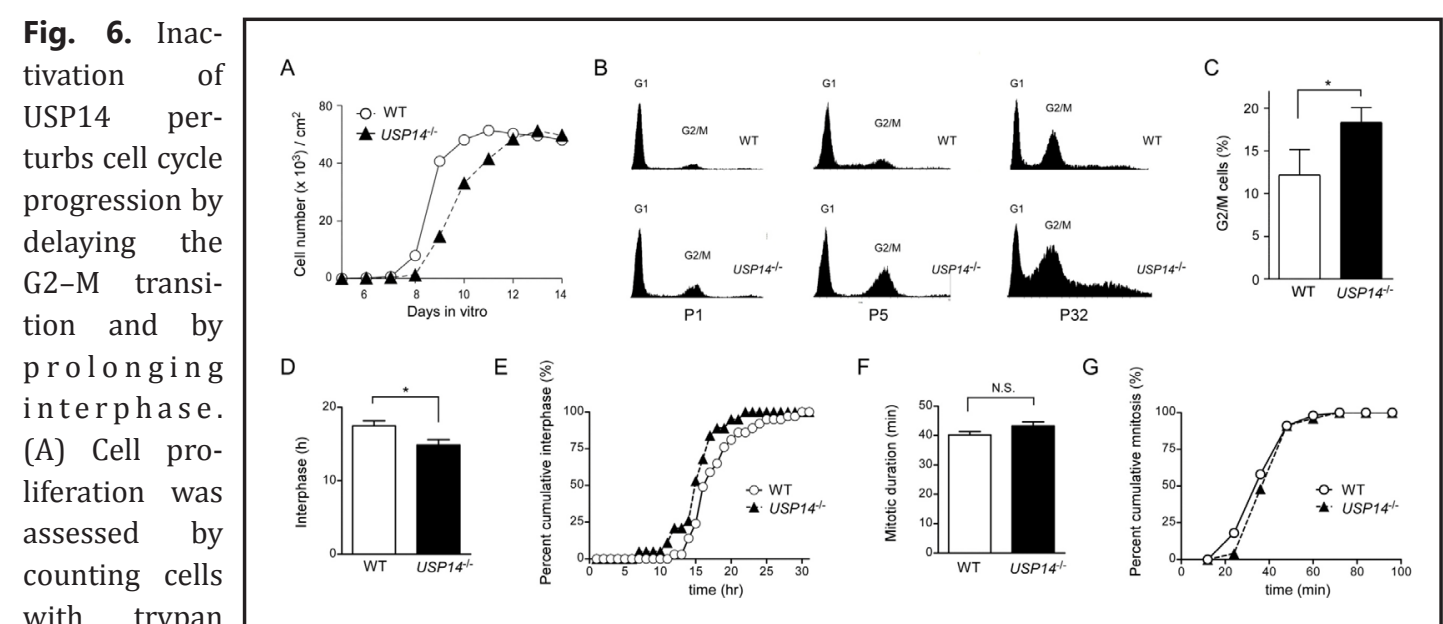

blue staining

on the indicated days after seeding. (B) Flow cytometry-based cell cycle analysis was performed by quantitation of DNA content with propidium iodide staining. Both primary (passage \#1 [P1] and passage \#5 [P5]) and immortalized WT and USP14 ${ }^{-/-}$MEFs were analyzed and compared. (C) The proportion of primary WT and USP14-/- MEFs at the G2-M transition. (D, E) Quantitative live-cell image analysis for assessing the duration of interphase (D) and its percentage cumulative curve (E). Cells were imaged for $48 \mathrm{~h}$ at 15-min time intervals. (F, G) Same as D and E, respectively, except that mitotic duration was analyzed in the same time-lapse images. Primary USP14 ${ }^{-/-}$MEFs showed significantly prolonged interphase, but mitotic time was not affected. For each condition, four random visual fields in three independent wells were imaged, and the results for the 12-image series were combined. Measured median values are provided; * $\mathrm{p}<0.05$ ( $\mathrm{n}=3$, twotailed Student's t test). N.S.: not statistically significant.

We next analyzed the role of USP14 in the formation of polyUb linkages by separating the endogenous polyUb conjugates by size. As shown in Fig. 5D, smeared bands of polyUbconjugates in a gel were separated into three regions such as high-molecular-weight (MW), middle-MW, and low-MW fractions, and were subjected to AQUA-MS. We found that the highMW and low-MW regions of gels contained greater amounts of total Ub in USP14 ${ }^{-{ }^{-}}$MEFs than in the WT (Fig. 5E). In the middle-MW region, however, total Ub amounts were slightly reduced in the USP14 knockout MEFs. The topologies of polyUb chains in these fractions were relatively similar although significantly greater amounts of chains based on either the Lys48 or Lys33 linkage were observed in the low-MW region (Fig. 5F). These results imply that USP14 is required for Ub chain trimming regardless of the topology of the chain in order for the substrate to be rescued from proteasomal degradation.

\section{The effect of USP14 deficiency on mammalian cell cycle progression}

To evaluate physiological consequences of USP14 deficiency for the cell, we used the immortalized MEFs to determine whether USP14 is implicated in cell proliferation. As shown in Fig. 6A, we found that the doubling time of USP14 $4^{-/-}$MEFs was significantly longer than that of WT MEFs. When analyzed by flow cytometry, this reduced proliferation of USP14null cells appeared to be associated with a greater proportion of cells arrested at the G2-M transition (Fig. 6B and 6C). The percentages of USP14 ${ }^{-/-}$MEFs with the cell cycle abnormality increased as a function of time as the cells were cultured in vitro: among the primary MEFs (P1), 20\% of USP14 ${ }^{-/-}$MEFs were at the G2-M transition, whereas only $12.2 \%$ of normal cells were in this phase. Among the immortalized USP14 ${ }^{-/}$MEFs, significantly higher percentages of cells were at the G2-M transition or even in aneuploid states as compared with WT MEFs (Fig. 6B and 6C). These results suggest that our finding in the immortalized USP14 ${ }^{-/-}$MEFs is unlikely to be a cellular artifact of the immortalization process because the delayed G2-M state was also observed in the cultured primary MEFs. It is also notable that the more cells in USP14 ${ }^{-/-}$MEFs at P32 were aneuploidic (Fig. 6C), possibly representing the 
adaptive response of immortalized cells under profound proteotoxic stress and relying on their increased proteasome activity for maintaining their proliferative states [38].

Next, we tried to measure the duration of interphase and mitosis using time-lapse images of cells expressing GFP-tagged histone 2B (H2B). Nuclei in the individual cells were tracked from frame to frame throughout cell division, and using an automated image analysis tool, key transition points could be identified as a function of time [24]. After infection of MEFs with a retrovirus expressing GFP-H2B, we found that USP14 $4^{-/-}$MEFs showed significantly shorter interphase than the WT did (median time of $14.9 \mathrm{~h}$ in USP14 ${ }^{-/-}$MEFs vs. $17.5 \mathrm{~h}$ in WT; Fig. 6D, 6E). There were no significant changes in mitosis duration (median time of 43.3 $\mathrm{h}$ in USP14 $1-$ MEFs vs. $40.2 \mathrm{~h}$ in WT; Fig. 6F and 6G) or the number of cells in the mitotic phase. Therefore, these findings further indicate that USP14 is required for a normal cell cycle especially at the G2-M transition rather than the mitotic processes.

To further elucidate whether the effect of USP14 inactivation is mediated by the G2-M delay, the cell cycle checkpoints, including amounts of cyclins A and B1, were examined by a reporter gene assay and IB analysis. In these experiments, WT or USP14 ${ }^{-/-}$MEFs were infected with an adenovirus expressing luciferase reporter genes containing the cyclin A or cyclin B1 promoter (cyclin A-Luc or cyclin B1-Luc, respectively), and the luciferase activity was compared between the MEF groups. As shown in Fig. 7A, USP14 ${ }^{-/-}$MEFs showed both elevated cyclin A-Luc and cyclin B1-Luc activity. Increased cyclin A and B1 reporter gene activities in USP14 ${ }^{-/-}$MEFs appeared to reflect increased protein levels (Fig. 7B). These results are consistent with our finding that USP14 $4^{-/-}$MEFs are abnormally G2-M arrested (Fig. 6C) because cyclin A is degraded in the late G2 phase and early mitotic phase, whereas cyclin B1 is degraded during mitotic exit. Thus, the elevated amounts of these cyclins may be related to the proliferation defect and a changed ubiquitome landscape in USP14 ${ }^{-/}$MEFs.

Because USP14 deficiency caused dramatic inhibition of cell growth by delaying cell cycle progression, we next tested whether USP14 inhibition can potentiate imatinib's antiproliferative effects on lymphocytes because it is known that the proteasome inhibitor carfilzomib acts synergistically with imatinib [39]. Treatment with IU1 alone showed a minimal effect on viability of Ba/F3 cells. In sharp contrast, treatment with IU1 in the presence of imatinib significantly increased toxicity to Ba/F3 cells (Fig. 7C), suggesting that the proteasome activator IU1 may be a promising therapeutic agent for chronic myeloid leukemia.

\footnotetext{
Fig. 7. The USP14 knockout led to elevated levels of cyclins $\mathrm{A}$ and $\mathrm{B}$, and USP14 i n h ib ition exerted cytotoxicity synergistically with

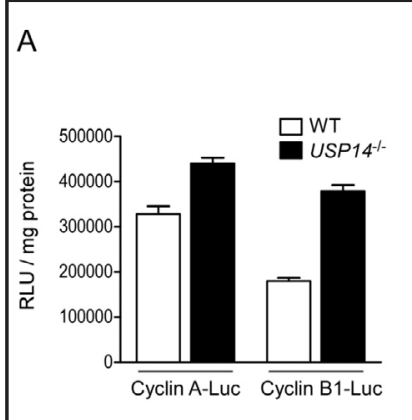

B

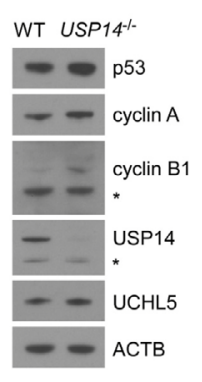
imatinib treatment. (A) A cyclin-luciferase activity assay indicated that immortalized USP14 ${ }^{-/-}$MEFs strongly expressed cyclins A and B as compared to WT MEFs. The cells were infected with adenoviruses expressing cyclin-luciferase fusion proteins under the control of cyclin promoters, and the luciferase activity was measured as a representative measure of cyclin levels. The data are presented as mean \pm SD from three independent experiments. RLU, relative luciferase unit. (B) WCEs from primary MEFs were analyzed by SDS-PAGE/IB with anti-cyclin A and anti-cyclin B antibodies. (C) The USP14 inhibitor IU1 showed synergistic (with imatinib) toxicity for Ba/F3 cells, which overexpress Bcr-Abl. Imatinib is a potent inhibitor of the Abl tyrosine kinase. These inhibitors were incubated with the cells for $24 \mathrm{~h}$ at indicated concentrations, and then an MTT assay was performed. Each data point represents mean \pm SD of three independent experiments. ${ }^{*} \mathrm{P}<0.05$ from Bonferroni's multiple comparison ANOVA test.
}

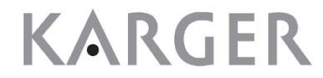




\section{Cellular Physiology and Biochemistry Published online: May 15, $2018 \quad \begin{aligned} & \text { DOI: 10.1159/000489750 } \\ & \text { www.karger.com/cpb }\end{aligned}$

The effect of USP14 deficiency on Drosophila cell cycle and eye development

To understand the regulatory roles of USP14 on cell cycle in vivo, we analyzed Drosophila eyes, which provides an excellent model for developmentally regulated cell proliferation [40]. First, to determine whether USP14 knockdown in vivo has any differences in the population of $\mathrm{S}$ and $\mathrm{M}$ phase cells, we examined the incorporation of bromodeoxyuridine (BrdU) and phospho-histone H3 (pH3) antibody labeling. We found overall similar levels and patterns of BrdUpositive nuclei in control (lacZ RNAi expressed in eye imaginal discs) and USP14 knockdown eye discs (Fig. 8A). However, we observed that the cells, especially under the second mitotic wave that are destined to enter the final round of division [41], showed significantly reduced pH3-positive signals (Fig. 8B). In addition, Drosophila eyes lacking USP14 showed a smaller number of mitotic cells behind the morphogenetic furrow than control flies, indicating less developing photoreceptors, along with delayed eye development and apparent morphological defects (Fig. 8B). Therefore, our data provide strong evidence that USP14 is

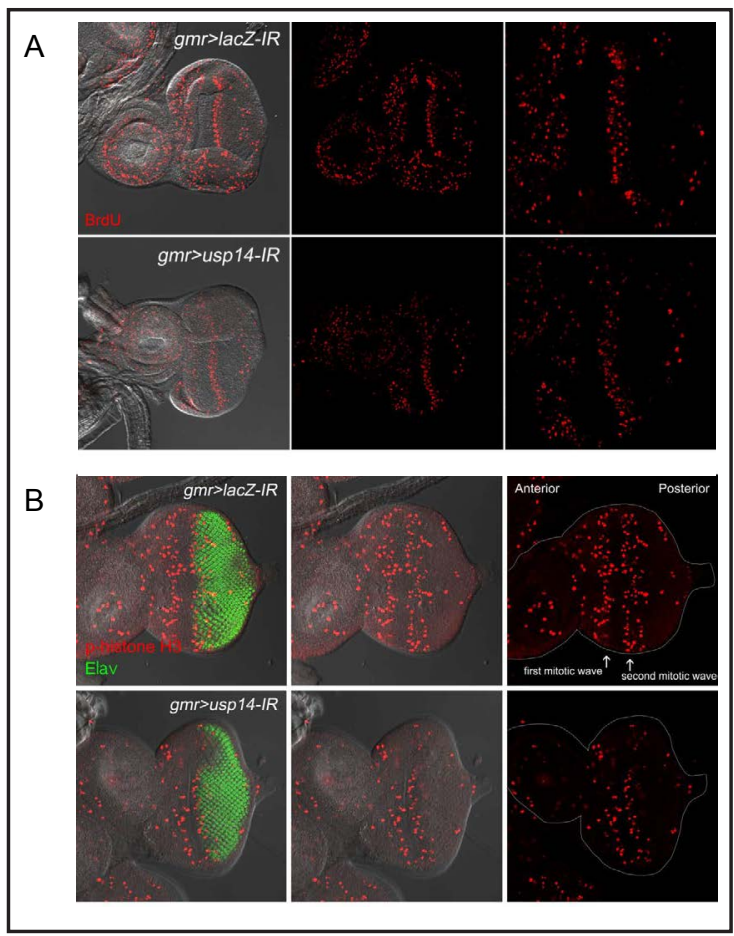

Fig. 8. Drosophila eyes lacking USP14 have developmental defects due to mitotic abnormality. (A) BrdU incorporation assay of transgenic Drosophila models expressing LacZ (gmr>lacZ-IR; upper panels) or USP14 (gmr>USP14>IR; lower) siRNA in larval eye imaginal disks. (B) As in A, except that the phospho-Histon H3 immunostaining was performed with Elav counterstaining. necessary for the maintenance of proper cell cycle regulation and development of Drosophila eyes.

\section{Discussion}

This study uncovered novel biological functions of USP14, which include the regulation of proteasome activity, cellular Ub homeostasis, and cell cycle progression. By characterizing primary and immortalized USP14 $4^{-/-}$MEFs, we demonstrated that USP14 deficiency elevates both proteasome activity and cellular DUB activity. The USP14 deficiency also perturbed ubiquitin homeostasis, leading to the shortage of free Ub pool in cells. In addition, the USP14 deficiency inhibited cell proliferation by extending the G2-M checkpoint, along with upregulation of cyclins A and B1. Also using genetic tools of Drosophila, we demonstrate the critical role of USP14 on Ub homeostasis and cell cycle regulation in vivo.

\section{Conclusion}

These cell physiological outcomes are expected to originate from complicated functions of USP14 on proteasomes, which include proteasome assembly, gate opening, catalytic or noncatalytic activity regulation, conformational state changes, and multiple polyUb chain preference. Our results should help to better understand complex regulatory functions of the proteasome that involve ubiquitin homeostasis and degradation of specific substrates related with various human diseases. 


\begin{tabular}{|c|c|c|}
\hline Cellular Physiology & Cell Physiol Biochem 2018; & $47: 67-82$ \\
\hline and Biochemistry & $\begin{array}{l}\text { DOI: } 10.1159 / 000489750 \\
\text { Published online: May 15, } 2018\end{array}$ & $\begin{array}{l}\text { O } 2018 \text { The Author(s). Published by S. Karger AG, Basel } \\
\text { www.karger.com/cpb }\end{array}$ \\
\hline
\end{tabular}

\section{Acknowledgements}

This work was supported by grants of the National Research Foundation (NRF) of Korea (2016R1C1B2011367 to J.H.L., 2017R1A6A3A11029936 to S.Y.P., 2015R1C1A2A01051560 to M.J.K., 2016R1A2B2006507 to M.J.L.), the Brain Research Program (2016M3C7A1913895 to M.J.L.), and the Creative-Pioneering Researchers Program of Seoul National University.

J.H.L. and S.P carried out most in vitro studies and cell-based assays. Y.Y and W.H.C. characterized proteasomes in MEFs. M.J.K. contributed to the Drosophila works. M.J.L. were responsible for the overall design of the project and manuscript preparation.

\section{Disclosure Statement}

The authors declare no Disclosure Statement.

\section{References}

1 Husnjak K, Elsasser S, Zhang N, Chen X, Randles L, Shi Y, Hofmann K, Walters KJ, Finley D, Dikic I: Proteasome subunit Rpn13 is a novel ubiquitin receptor. Nature 2008;453:481-488.

-2 Shi Y, Chen X, Elsasser S, Stocks BB, Tian G, Lee BH, Zhang N, de Poot SA, Tuebing F, Sun S, Vannoy J, Tarasov SG, Engen JR, Finley D, Walters KJ: Rpn1 provides adjacent receptor sites for substrate binding and deubiquitination by the proteasome. Science 2016;351:aad9421

3 Komander D: The emerging complexity of protein ubiquitination. Biochem Soc Trans 2009;37:937-953.

4 Ikeda F, Dikic I: Atypical ubiquitin chains: new molecular signals. 'Protein Modifications: Beyond the Usual Suspects' review series. EMBO Rep 2008;9:536-542.

5 Kirkpatrick DS, Hathaway NA, Hanna J, Elsasser S, Rush J, Finley D, King RW, Gygi SP: Quantitative analysis of in vitro ubiquitinated cyclin B1 reveals complex chain topology. Nat Cell Biol 2006;8:700-710.

6 Bish RA, Fregoso OI, Piccini A, Myers MP: Conjugation of complex polyubiquitin chains to WRNIP1. J Proteome Res 2008;7:3481-3489.

7 Grasso G, Santoro AM, Lanza V, Sbardella D, Tundo GR, Ciaccio C, Marini S, Coletta M, Milardi D: The double faced role of copper in A beta homeostasis: A survey on the interrelationship between metal dyshomeostasis, UPS functioning and autophagy in neurodegeneration. Coordination Chemistry Reviews 2017;347:1-22.

-8 Jiang Y, Lee J, Lee JH, Lee JW, Kim JH, Choi WH, Yoo YD, Cha-Molstad H, Kim BY, Kwon YT, Noh SA, Kim KP, Lee MJ: The arginylation branch of the N-end rule pathway positively regulates cellular autophagic flux and clearance of proteotoxic proteins. Autophagy 2016;12:2197-2212.

-9 Lee MJ, Lee BH, Hanna J, King RW, Finley D: Trimming of ubiquitin chains by proteasome-associated deubiquitinating enzymes. Mol Cell Proteomics 2011;10:R110 003871.

10 de Poot SAH, Tian G, Finley D: Meddling with Fate: The Proteasomal Deubiquitinating Enzymes. J Mol Biol 2017;429:3525-3545.

11 Hanna J, Hathaway NA, Tone Y, Crosas B, Elsasser S, Kirkpatrick DS, Leggett DS, Gygi SP, King RW, Finley D: Deubiquitinating enzyme Ubp6 functions noncatalytically to delay proteasomal degradation. Cell 2006;127:99-111.

12 Baboshina OV, Crinelli R, Siepmann TJ, Haas AL: N-end rule specificity within the ubiquitin/proteasome pathway is not an affinity effect. J Biol Chem 2001;276:39428-39437.

-13 Mansour W, Nakasone MA, von Delbruck M, Yu Z, Krutauz D, Reis N, Kleifeld O, Sommer T, Fushman D, Glickman MH: Disassembly of Lys11 and mixed linkage polyubiquitin conjugates provides insights into function of proteasomal deubiquitinases Rpn11 and Ubp6. J Biol Chem 2015;290:4688-4704.

14 Choi WH, de Poot SA, Lee JH, Kim JH, Han DH, Kim YK, Finley D, Lee MJ: Open-gate mutants of the mammalian proteasome show enhanced ubiquitin-conjugate degradation. Nat Commun 2016;7:10963.

15 Han DH, Na HK, Choi WH, Lee JH, Kim YK, Won C, Lee SH, Kim KP, Kuret J, Min DH, Lee MJ: Direct cellular delivery of human proteasomes to delay tau aggregation. Nat Commun 2014;5:5633.

-16 Bashore C, Dambacher CM, Goodall EA, Matyskiela ME, Lander GC, Martin A: Ubp6 deubiquitinase controls conformational dynamics and substrate degradation of the 26S proteasome. Nat Struct Mol Biol 2015;22:712-719.

17 Santoro AM, Monaco I, Attanasio F, Lanza V, Pappalardo G, Tomasello MF, Cunsolo A, Rizzarelli E, De Luigi A, Salmona M, Milardi D: Copper(II) ions affect the gating dynamics of the 20S proteasome: a molecular and in cell study. Sci Rep 2016;6:33444. 


\section{Cellular Physiology Cell Physiol Biochem 2018;47:67-82 \begin{tabular}{l|l} 
and Biochemistry Published online: May 15, 2018 & $\begin{array}{l}\text { D } 2018 \text { The Author(s). Published by S. Karger AG, Basel } \\
\text { www.karger.com/cpb }\end{array}$ \\
\hline
\end{tabular}}

Lee et al.: Cellular and Biochemical Roles of USP14

18 Arena G, Bellia F, Frasca G, Grasso G, Lanza V, Rizzarelli E, Tabbi G, Zito V, Milardi D: Inorganic stressors of ubiquitin. Inorg Chem 2013;52:9567-9573.

19 Choi TS, Lee J, Han JY, Jung BC, Wongkongkathep P, Loo JA, Lee MJ, Kim HI: Supramolecular Modulation of Structural Polymorphism in Pathogenic alpha-Synuclein Fibrils Using Cu(II) Coordination. Angew Chem Int Ed Engl 2018;57:3099-3103.

20 Shin SK, Kim JH, Lee JH, Son YH, Lee MW, Kim HJ, Noh SA, Kim KP, Kim IG, Lee MJ: Docosahexaenoic acidmediated protein aggregates may reduce proteasome activity and delay myotube degradation during muscle atrophy in vitro. Exp Mol Med 2017;49:e287.

-21 Kim W, Bennett EJ, Huttlin EL, Guo A, Li J, Possemato A, Sowa ME, Rad R, Rush J, Comb MJ, Harper JW, Gygi SP: Systematic and quantitative assessment of the ubiquitin-modified proteome. Mol Cell 2011;44:325340.

22 Lee MJ, Kim DE, Zakrzewska A, Yoo YD, Kim SH, Kim ST, Seo JW, Lee YS, Dorn GW, 2nd, Oh U, Kim BY, Kwon YT: Characterization of arginylation branch of $\mathrm{N}$-end rule pathway in G-protein-mediated proliferation and signaling of cardiomyocytes. J Biol Chem 2012;287:24043-24052.

-23 Kim E, Kim S, Lee JH, Kwon YT, Lee MJ: Ablation of Arg-tRNA-protein transferases results in defective neural tube development. BMB Rep 2016;49:443-448.

24 Sigoillot FD, Huckins JF, Li F, Zhou X, Wong ST, King RW: A time-series method for automated measurement of changes in mitotic and interphase duration from time-lapse movies. PLoS One 2011;6:e25511.

25 Zhang GJ, Chen TB, Davide J, Tao W, Vanko A, Connolly B, Williams DL, Jr., Sur C: Visualization of mitotic arrest of cell cycle with bioluminescence imaging in living animals. Mol Imaging Biol 2013;15:431-440.

-26 Brand AH, Perrimon N: Targeted gene expression as a means of altering cell fates and generating dominant phenotypes. Development 1993;118:401-415.

27 Kim JH, Kim E, Choi WH, Lee J, Lee JH, Lee H, Kim DE, Suh YH, Lee MJ: Inhibitory RNA Aptamers of Tau Oligomerization and Their Neuroprotective Roles against Proteotoxic Stress. Mol Pharm 2016;13:20392048.

28 Lee BH, Lee MJ, Park S, Oh DC, Elsasser S, Chen PC, Gartner C, Dimova N, Hanna J, Gygi SP, Wilson SM, King RW, Finley D: Enhancement of proteasome activity by a small-molecule inhibitor of Usp14. Nature 2010;467:179-184.

29 Crimmins S, Sutovsky M, Chen PC, Huffman A, Wheeler C, Swing DA, Roth K, Wilson J, Sutovsky P, Wilson S: Transgenic rescue of ataxia mice reveals a male-specific sterility defect. Dev Biol 2009;325:33-42.

-30 Kim HT, Goldberg AL: The deubiquitinating enzyme Usp14 allosterically inhibits multiple proteasomal activities and ubiquitin-independent proteolysis. J Biol Chem 2017;292:9830-9839.

-31 Kuo CL, Goldberg AL: Ubiquitinated proteins promote the association of proteasomes with the deubiquitinating enzyme Usp14 and the ubiquitin ligase Ube3c. Proc Natl Acad Sci U S A 2017;114:E3404-E3413.

-32 Sakata E, Stengel F, Fukunaga K, Zhou M, Saeki Y, Forster F, Baumeister W, Tanaka K, Robinson CV: The catalytic activity of Ubp6 enhances maturation of the proteasomal regulatory particle. Mol Cell 2011;42:637-649.

33 Peth A, Besche HC, Goldberg AL: Ubiquitinated proteins activate the proteasome by binding to Usp14/ Ubp6, which causes 20S gate opening. Molecular Cell 2009;36:794-804.

34 Hanna J, Meides A, Zhang DP, Finley D: A ubiquitin stress response induces altered proteasome composition. Cell 2007;129:747-759.

35 Jung H, Kim BG, Han WH, Lee JH, Cho JY, Park WS, Maurice MM, Han JK, Lee MJ, Finley D, Jho EH: Deubiquitination of Dishevelled by Usp14 is required for Wnt signaling. Oncogenesis 2013;2:e64.

-36 Demontis F, Perrimon N: FOXO/4E-BP signaling in Drosophila muscles regulates organism-wide proteostasis during aging. Cell 2010;143:813-825.

37 Lee BH, Lu Y, Prado MA, Shi Y, Tian G, Sun S, Elsasser S, Gygi SP, King RW, Finley D: USP14 deubiquitinates proteasome-bound substrates that are ubiquitinated at multiple sites. Nature 2016;532:398-401.

-38 Torres EM, Dephoure N, Panneerselvam A, Tucker CM, Whittaker CA, Gygi SP, Dunham MJ, Amon A: Identification of aneuploidy-tolerating mutations. Cell 2010;143:71-83.

-39 Crawford LJ, Chan ET, Aujay M, Holyoake TL, Melo JV, Jorgensen HG, Suresh S, Walker B, Irvine AE: Synergistic effects of proteasome inhibitor carfilzomib in combination with tyrosine kinase inhibitors in imatinib-sensitive and -resistant chronic myeloid leukemia models. Oncogenesis 2014;3:e90.

40 Kang MJ, Vasudevan D, Kang K, Kim K, Park JE, Zhang N, Zeng X, Neubert TA, Marr MT, 2nd, Ryoo HD: 4E-BP is a target of the GCN2-ATF4 pathway during Drosophila development and aging. J Cell Biol 2017;216:115129.

41 Baker NE: Cell proliferation, survival, and death in the Drosophila eye. Semin Cell Dev Biol 2001;12:499507. 